Pacific Journal of Mathematic 


\title{
ON A CALCULUS OF PARTITION FUNCTIONS
}

\author{
GEORGE E. ANDREWS
}

The main object in this paper is to show that many partition theorems which have been deduced from identities in basic hypergeometric series and infinite products may in fact be given purely combinatorial proofs. We show that the manipulations performed on the generating functions have combinatorial interpretations, and thus we obtain a "calculus of partition functions" which translates a sizable portion of the techniques of the elementary theory of basic hypergeometric series into arithmetic terms.

In [13], Vahlen derived a large number of partition theorems combinatorially. Some of his initial results are actually arithmetic proofs of simple infinite product identities. For example, his derivation of equation (10) $[13 ;$ p. 4] is an arithmetic proof of

$$
\frac{\prod_{j=1}^{\infty}\left(1-q^{j}\right)}{\prod_{h=1}^{\infty}\left(1-q^{h}\right)}=1 .
$$

In $\S 2$, we shall extend the results of Vahlen (Lemmas 3, 4, and 5) and derive some further arithmetic proofs of well-known identities. These results will form the basis of our calculus. In $\S 3$, we illustrate the use of our calculus by giving new combinatorial proofs of Euler's theorem [9; p. 277, Th. 344] and Jacobi's identity [9; p. 282].

It should be stressed that the interest of these results lies not so much in their contribution to the search for new partition theorems as in their clarification of the relationship between combinatorial partition theory and what was previously the purely analytic aspect of partition theory. Thus we include in $\S 3$ only two results of a rather simple character; even these are relatively complicated to prove by our calculus. However, the method of proof is equally applicable to all the analytic results in [2], [3], [4], [5]; in such results Lemma 2 is crucial. And indeed the result on the order of a partition in [8] indicates that one of Rogers's proofs of the Rogers-Ramanujan identities may now be translated into a combinatorial proof.

2. Fundamental lemmas. We let $\sum$ denote the set of all doubly infinite sequences of nonnegative integers $\left\{f_{n}\right\}_{n=-\infty}^{\infty}=\left\{f_{n}\right\}$ for which $f_{n}=0$ for all but finitely many $n$. We define a partition condition $R$ to be a subset of $\sum$. We say that a partition $n$ of the 
form $n=b_{1}+b_{2}+\cdots=\sum_{i=-\infty}^{\infty} f_{i} \cdot i$ (where $f_{i}$ is the number of times $i$ appears as a summand) satisfies the condition $R$ if $\left\{f_{i}\right\} \in R$. Thus if $R=\left[\left\{f_{i}\right\} \mid f_{2 j}=0\right.$ for all $j$, and $f_{i}=0$ for $\left.i \leqq 0\right\}$, then $R$ is just the condition that the partition have only odd parts $(>0)$. The symbol $\pi$ will denote a finite set of integers (also $\pi$ may denote the sum of this set; no confusion should arise however), and the notation $\pi \in R$ means that if we write $\pi=\sum_{i=-\infty}^{\infty} f_{i} \cdot i$ then $\left\{f_{i}\right\} \in R$. If $\pi=$ $\sum f_{i} \cdot i$, then $\sharp \pi=\sum f_{i}$, and $\#^{\prime} \pi$ denotes the number of $i$ for which $f_{i} \neq 0$. Next if $R$ is a partition condition then $R^{d}$ is the subset of $R$ defined by $R^{d}=\left\{\left\{f_{i}\right\} \mid\left\{f_{i}\right\} \in R, f_{i} \leqq 1\right\}$. We also introduce a symbol which is essentially used by Vahlen $[13 ;$ p.2] for his treatment of partition functions; namely, $N(s=\pi, \pi \in R)$ denotes the number of partitions of $s$ which satisfy the condition $R$. Sometimes we may count partitions of $s$ utilizing a weighting factor $\omega_{\pi}$ (e.g., $\omega_{\pi}$ might be $(-1)^{\sharp \pi}$ or $\left.(-1)^{\sharp / \pi}\right)$; we denote this count by $N\left(s=\pi, \pi \in R ; \omega_{\pi}\right)$. We may also wish to count partitions with compound partition conditions; for example $N\left(s=\pi+\pi^{*}, \pi \in R_{1}, \pi^{*} \in R_{2}\right)$ denotes the number of partitions of $s$ of the form $s=\sum_{i=-\infty}^{\infty} f_{i} \cdot i+\sum_{j=-\infty}^{\infty} f_{j}^{\prime} \cdot j$, where $\left\{f_{i}\right\} \in R_{1},\left\{f_{j}^{\prime}\right\} \in R_{2}$. Further we let $\mathscr{C}_{r}\left(\alpha_{1}, \cdots, \alpha_{j} ; k\right)=\left[\left\{f_{i}\right\} \mid f_{i}=0\right.$ for $i \leqq r-1$, and $f_{i}=0$ unless $\left.i \equiv \alpha_{1}, \alpha_{2}, \cdots, \alpha_{j}(\bmod k)\right]$; for simplicity $\mathscr{Z}_{r}=\mathscr{C}_{r}(1 ; 1), \mathscr{U}_{r}=\mathscr{C}_{r}(1 ; 2), \mathscr{C}_{r}=\mathscr{C}_{r}(2 ; 2)$. Finally $l(\pi)$ denotes the largest part appearing in $\pi$.

Our first lemma is the arithmetic equivalent of multiplication of generating functions.

Lemma 1. Suppose for all $s$,

$$
\begin{aligned}
& N\left(s=\pi, \pi \in R_{1} ; \omega_{\pi}\right)=N\left(s=\pi, \pi \in R_{2}, \tilde{\omega}_{\pi}\right) \\
& N\left(s=\pi^{\prime}, \pi^{\prime} \in R_{1}^{\prime} ; \sigma_{\pi^{\prime}}\right)=N\left(s=\pi^{\prime}, \pi^{\prime} \in R_{2}^{\prime} ; \widetilde{\sigma}_{\pi^{\prime}}\right) .
\end{aligned}
$$

Then for all s,

$$
\begin{aligned}
N(s & \left.=\pi+\pi^{\prime}, \pi \in R_{1}, \pi^{\prime} \in R_{1}^{\prime} ; \omega_{\pi} \sigma_{\pi^{\prime}}\right) \\
& =N\left(s=\pi+\pi^{\prime}, \pi \in R_{2}, \pi^{\prime} \in R_{2}^{\prime} ; \tilde{\omega}_{\pi} \widetilde{\sigma}_{\pi^{\prime}}\right) .
\end{aligned}
$$

Proof.

$$
\begin{aligned}
N(s & \left.=\pi+\pi^{\prime}, \pi \in R_{1}, \pi^{\prime} \in R_{1}^{\prime} ; \omega_{\pi} \sigma_{\pi^{\prime}}\right) \\
& =\sum_{\substack{\pi \in R_{1} \\
\pi^{\prime} \in R_{1}^{\prime} \\
s=\pi+\pi^{\prime}}} \omega_{\pi} \sigma_{\pi^{\prime}}=\sum_{\pi \in R_{1}} \omega_{\pi} \sum_{\substack{\pi^{\prime} \in R_{1}^{\prime} \\
s-\pi=\pi^{\prime}}} \sigma_{\pi^{\prime}} \\
& =\sum_{\pi \in R_{1}} \omega_{\pi} \sum_{\substack{\pi^{\prime} \in R_{2}^{\prime} \\
s-\pi=\pi^{\prime}}} \widetilde{\sigma}_{\pi^{\prime}}
\end{aligned}
$$




$$
\begin{aligned}
& =\sum_{\substack{\pi \in R_{1} \\
\pi^{\prime} \in R_{2}^{\prime} \\
s=\pi+\pi^{\prime}}} \omega_{\pi} \widetilde{\sigma}_{\pi^{\prime}}=\sum_{\pi^{\prime} \in R_{2}^{\prime}} \widetilde{\sigma}_{\pi^{\prime}} \sum_{\substack{\pi \in R_{1} \\
s-\pi^{\prime}=\pi}} \omega_{\pi} \\
& =\sum_{\pi^{\prime} \in R_{2}^{\prime}} \widetilde{\sigma}_{\pi^{\prime}} \sum_{\substack{\pi \in R_{2} \\
s-\pi^{\prime}=\pi}} \tilde{\omega}_{\pi}=\sum_{\substack{\pi \in R_{2} \\
\pi^{\prime} \in R_{2}^{\prime} \\
s=\pi+\pi^{\prime}}} \tilde{\omega}_{\pi} \widetilde{\sigma}_{\pi^{\prime}} \cdot
\end{aligned}
$$

Our next result is the arithmetic equivalent of $[10 ;$ p. 92, e.q., (3. 2. 2.12)]

$$
{ }_{1} \phi_{0}[a ; z ; q] \equiv 1+\sum_{n=1}^{\infty} \frac{(1-a) \cdots\left(1-a q^{n-1}\right) z^{n}}{(1-q) \cdots\left(1-q^{n}\right)}=\prod_{j=0}^{\infty} \frac{\left(1-a z q^{j}\right)}{\left(1-z q^{j}\right)} .
$$

An arithmetic proof of (2.1) previously appeared in [6; §2]; however the proof here is more natural in that it involves only ordinary partitions and the one-to-one correspondence established is between partitions not sets of partitions of equal cardinality. The technique of Lemma 2 is used to prove a different partition theorem in [7].

Lemma 2. Let $m \geqq 0, n \geqq 0,1 \leqq \alpha<\beta$ be fixed integers. Then

$$
\begin{aligned}
N(s & \left.=\pi+\pi^{\prime}, \pi \in \mathscr{C}_{\alpha+1}(\alpha ; \beta)^{d}, \pi^{\prime} \in \mathscr{C}_{1}(\beta ; \beta), \sharp \pi+\sharp \pi^{\prime}=n, \sharp \pi=m\right) \\
& =N\left(s=\pi+\pi^{\prime}, \pi \in \mathscr{C}_{1}(\alpha ; \beta)^{d}, \pi^{\prime} \in \mathscr{C}_{1}(\beta ; \beta), l\left(\pi^{\prime}\right)=\beta n, \sharp \pi=m\right) .
\end{aligned}
$$

Proof. We establish a one-to-one correspondence between the sets of partitions described above. Let $\pi+\pi^{\prime}$ be any partition of the type enumerated by the right-hand side of the above equation. We write $\pi$ graph-theoretically as follows. Each part of the form $\beta \nu$ contributes $\beta$ rows of $\nu$ dots, and each part of the form $\beta \nu+\alpha$ contributes $\alpha$ rows of $(\nu+1)$ dots and $(\beta-\alpha)$ rows of $\nu$ dots. Now consider the partition obtained from the columns of the above-mentioned representation of the partition. It is clear that all parts are $\equiv 0, \alpha(\bmod \beta)$ and there are again $m$ parts of the form $\beta \nu+\alpha$ although now $\alpha$ cannot appear as a summand; also now there are exactly $n$ parts appearing. Thus we have a partition of the type enumerated by the left-hand side of the above equation. The above procedure is reversible and thus establishes a one-to-one correspondence between the two sets of partitions enumerated by the given partition functions. This establishes Lemma 2.

We now prove three lemmas which are generalizations of results due to Vahlen. The proofs are all similar so we give the details only for Lemma 3 (c.f. [13; p. 5, eq., (14)]). The proof of Lemma 4 is similar and may easily be obtained by proper extension of Vahlen's proof of his equation (13) $[13 ;$ p. 5]. Lemma 5 is in the same vein. 
Lemma 3. Let $\eta$ be any set of integers bounded below. Let, $2 \eta=\{n \mid n=2 m, m \in \eta\}$, and let $R(\eta)=\left\{\left\{f_{i}\right\} \mid f_{i}=0\right.$ if $\left.i \notin \eta\right\}$. Then

$$
\begin{aligned}
N(s & \left.=\pi+\pi^{\prime}, \pi \& \pi^{\prime} \in R(\eta)^{d} ; \sharp \pi+\# \pi^{\prime}=t ;(-1)^{\sharp^{-}}\right) \\
& = \begin{cases}(-1)^{t / 2} N\left(s=\pi, \pi \in R(2 \eta)^{d}, \sharp \pi=t / 2\right) \text { t even } \\
0 & t \text { odd } .\end{cases}
\end{aligned}
$$

REMARK. Since $\eta$ is bounded below both sides of the above equation are finite.

Proof. Let $\pi+\pi^{\prime}$ be a partition of the type enumerated by the lefthand side of the above equation. Suppose there are exactly $\nu(>0)$ numbers which are summands of either $\pi$ or $\pi^{\prime}$ but not both. The remaining summands appear once in $\pi$ and once in $\pi^{\prime}$.

Thus given the totality of summands of $\pi+\pi^{\prime}$ we see that many different partitions of the type enumerated by the left-hand side of the above equation may be formed; indeed pick any subset of the $\nu$ distinct parts to form a portion of $\pi$, put the remainder of the $\nu$ parts in $\pi^{\prime}$ and split the repeated parts between $\pi$ and $\pi^{\prime}$. Thus there are

$$
\left(\begin{array}{l}
\nu \\
0
\end{array}\right)+\left(\begin{array}{l}
\nu \\
2
\end{array}\right)+\left(\begin{array}{l}
\nu \\
4
\end{array}\right)+\cdots=2^{\nu-1}
$$

partitions formed with $\sharp \pi-\frac{1}{2}(t-\nu)$ even, and

$$
\left(\begin{array}{l}
\nu \\
1
\end{array}\right)+\left(\begin{array}{l}
\nu \\
3
\end{array}\right)+\left(\begin{array}{l}
\nu \\
5
\end{array}\right)+\cdots=2^{\nu-1}
$$

partitions formed with $\sharp \pi-\frac{1}{2}(t-\nu)$, odd. Thus counting with weight $(-1)^{*-\pi}$ we see that as long as $\nu>0$ we have zero total contribution to our count. Thus

$$
\begin{aligned}
N(s & \left.=\pi+\pi^{\prime}, \pi \in R(\eta)^{d}, \sharp \pi+\sharp \pi^{\prime}=t ;(-1)^{\sharp-}\right) \\
& =\left\{\begin{array}{l}
N\left(s=\pi+\pi, \pi \in R(\eta)^{d}, \sharp \pi=t / 2 ;(-1)^{\sharp-}\right), t \text { even } \\
0, t \text { odd. }
\end{array}\right. \\
& =\left\{\begin{array}{l}
(-1)^{t / 2} N\left(s=\pi, \pi \in R(2 \eta)^{d}, \sharp \pi=t / 2\right), t \text { even } \\
0, t \text { odd. }
\end{array}\right.
\end{aligned}
$$

This concludes the proof of Lemma 3 .

Our next lemma treats

$$
\prod_{j \in \mathscr{S}}\left(1-a q^{j}\right)^{-1}=\prod_{k \in \mathscr{S}-\mathscr{S}^{\infty}}\left(1-a q^{k}\right) / \prod_{h \in \mathscr{S}}\left(1-a q^{h}\right),
$$

where $\mathscr{S}$ and $\mathscr{T}$ denote any two sets of integers $(\geqq M)$ such that $\mathscr{S} \subseteq \mathscr{T}$. 
Lemma 4.

$$
\begin{aligned}
N(s= & \pi, \pi \in R(\mathscr{S}), \sharp \pi=m) \\
= & N\left(s=\pi+\pi^{\prime}, \pi \in R(\mathscr{S}-\mathscr{S})^{d}, \pi^{\prime} \in R(\mathscr{T}), \sharp \pi+\sharp \pi^{\prime}\right. \\
& \left.=m ;(-1)^{\sharp \pi}\right) .
\end{aligned}
$$

Proof. The proof is very similar to that of Lemma 3 and is given in detail by Vahlen when $\mathscr{S}$ is empty, $m$ is arbitrary, and $\mathscr{T}$ is the set of positive integers.

Our final lemma arithmetizes the reciprocal identity of (2.2).

Lemma 5. Let. $\mathscr{S} \cong \mathscr{T}$ denote any two sets of integers $(\geqq M)$. Then

$$
\begin{gathered}
N\left(s=\pi, \pi \in R(\mathscr{P})^{d}, \sharp \pi=m\right)=N\left(s=\pi+\pi^{\prime}, \pi \in R(\mathscr{T})^{d},\right. \\
\left.\pi^{\prime} \in R(\mathscr{S}-\mathscr{P}), \sharp \pi+\mathbb{N}^{\prime}=m ;(-1)^{\sharp n^{\prime}}\right) .
\end{gathered}
$$

3. Partition function identities. The technique here is conceptually very simple. We take a result from the elementary theory of basic hypergeometric series and infinite products and then translate the steps of the proof into arithmetic terms utilizing Lemmas 1-5.

We start with a well-known theorem of Euler.

THEOREM 1. Let $(n)$ denote the number of partitions of $n$ into odd parts. Let $\mathscr{Q}(n)$ denote the number of partitions of $n$ into distinct parts. Then $\mathscr{O}(n)=Q(n)$.

REMARK. Arithmetic proofs of this theorem already exist $[12 ; \mathrm{p}$. 45]. Indeed Vahlen gives an arithmetic proof $[13 ; \mathrm{p} .3]$ which is altogether different from the following.

Proof. We shall arithmetize the following identities

$$
\prod_{j=1}^{\infty}\left(1+q^{j}\right)=\prod_{j=1}^{\infty}\left(1-q^{2 j}\right) /\left(1-q^{j}\right)=\prod_{j=0}^{\infty}\left(1-q^{2 j+1}\right)^{-1} .
$$

First $\mathscr{O}(n)=N\left(s=\pi, \pi \in \mathscr{Z}_{1}\right)$, and $\mathscr{Q}(n)=N\left(s=\pi, \pi \in \mathscr{C}_{1}^{d}\right)$. Now

$$
\begin{aligned}
& N\left(s=\pi, \pi \in x_{1}^{\prime}\right) \\
& =N\left(s=\pi+\pi^{\prime}+\pi^{\prime \prime}, \pi \text { and } \pi^{\prime} \in \mathscr{Q}_{1}^{d}, \pi^{\prime \prime} \in \gamma_{1}^{\prime} ;(-1)^{\sharp \pi^{\prime}}\right) \\
& \text { (by Lemmas } 1 \text { and 4) } \\
& =N\left(s=\pi+\pi^{\prime \prime}, \pi \in \mathscr{C}^{d}, \pi^{\prime \prime} \in \mathscr{K}_{1} ;(-1)^{\sharp *}\right)(\text { by Lemmas } 1 \text { and } 3) \\
& =N\left(s=\pi, \pi \in \mathscr{C}_{1}\right) \\
& \text { (by Lemma 4). } \\
& =\mathscr{O}(n) \text {. }
\end{aligned}
$$


The above is shorthand for the following combinatorial processes. We write

$$
\begin{aligned}
& \sum_{i=1}^{r} a_{i}+\sum_{j=1}^{s} b_{j}+\sum_{k=1}^{t} c_{k}= \\
& a_{1}+a_{2}+\cdots+a_{r} / b_{1}+b_{2}+\cdots+b_{s} / c_{1}+c_{2}+\cdots+c_{t} .
\end{aligned}
$$

Let us consider the partitions of 3 into distinct parts namely 3 and $2+1$; thus $\mathbb{Q}(3)=2$. The second expression in the above proof counts the excess of the 9 partitions $3 / /_{-},-/_{-} / 3,2+1 / /_{-},-/ 2+1 /{ }_{-}, /_{-} / 2+1$, $2 / \_/ 1,1 / /_{-} / 2,1 / /_{-} / 2,1 /{ }_{-} / 1+1,{ }_{-} / / 1+1+1$ over the 7 partitions $\_/ 3 /-$, $2 / 1 /_{-}, 1 / 2 / /_{-} / 2 / 1,1_{-} / 1 / 2,1 / 1 / 1,-/ 1 / 1+1$. The pairing described in Lemma 4 to insure this excess is still 2 is as follows.

$$
\begin{aligned}
& 3 /-1 \\
& 2+1 / / / \\
& -/-/ 3 \leftrightarrow-/ 3 /-
\end{aligned}
$$

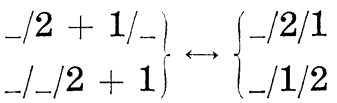

$$
\begin{aligned}
& 2 / \_/ 1 \quad \leftrightarrow 2 / 1 / \_ \\
& 1 / / 2 \leftrightarrow 1 / 2 / \_ \\
& 1 / / 1+1 \leftrightarrow 1 / 1 / 1 \\
& -/-/ 1+1+1 \leftrightarrow-/ 1 / 1+1 .
\end{aligned}
$$

The third expression counts the excess of the 3 partitions $-/ 3$, $-/ 2+1,-/ 1+1+1$, over the single partition $2 / 1$. The previous sets of partitions are now paired in a new manner as described in Lemma 3 to accomplish this, namely

$$
\begin{aligned}
& 3 /-/-\quad \leftrightarrow-/ 3 / \\
& 2+1 /-/-1 \leftrightarrow\left\{\begin{array}{l}
2 / 1 /- \\
1 / 2 / /
\end{array}\right. \\
& -/ 2+1 /\}_{-} \leftrightarrow 1 / 2 / \ldots \\
& 2 /-/ 1 \quad \leftrightarrow{ }_{-} / 2 / 1 \\
& 1 / \_/ 2 \quad \leftrightarrow \ldots / 1 / 2 \\
& 1 / \_/ 1+1 \leftrightarrow{ }_{-} / 1 / 1+1 \\
& -/-/ 3 \\
& -/-/ 2+1 \\
& -/-1+1+1 \\
& 1 / 1 / 1 \text {. }
\end{aligned}
$$

The first two partitions of the unpaired partitions above are added to yield ${ }_{-} / 3,_{-} / 2+1$, and $-/ 1+1+1$ in the left column and $2 / 1$ in the right column.

Finally these partitions are paired according to Lemma 4. Namely, 


$$
\begin{aligned}
& -/ 3 \\
& -/ 1+1+1 \\
& -/ 2+1 \leftrightarrow 2 / 1 .
\end{aligned}
$$

This leaves the two partitions of 3 into odd parts, viz. $3,1+1+1$.

Next we give a proof of Jacobi's triple product identity [9; $\mathrm{p}$. 282]. Previous arithmetic proofs have been given by Sylvester [12; p. 34-36], Vahlen [13; p. 10-12], Wright [14], and Sudler [11]. This proof is the arithmetization of the proof appearing in [1].

Our result will prove Jacobi's identity in the form

$$
\prod_{j=1}^{\infty}\left(1-q^{2 j}\right)\left(1+z q^{2 j-1}\right)=\sum_{-\infty}^{\infty} q^{n^{2}} z^{n} \cdot \prod_{j=1}^{\infty}\left(1+z^{-1} q^{2 j-1}\right)^{-1} .
$$

THEOREM 2.

$$
\begin{aligned}
N(s & \left.=\pi+\pi^{\prime} ; \pi \in \mathscr{S}_{1}^{d}, \pi^{\prime} \in \mathscr{U}_{1}^{d}, \# \pi^{\prime}=m ;(-1)^{\# \pi}\right) \\
& =N\left(s=n^{2}+\pi, \pi \in \mathscr{U}_{1}, n-\# \pi=m\right.
\end{aligned}
$$

( $n$ an arbitrary integer); $\left.(-1)^{\sharp \pi}\right)$.

Proof. Let $m$ and $s$ be fixed integers $s \geqq 0$. First if $m \geqq 0$

$$
\begin{aligned}
N(s= & \left.\pi+\pi^{\prime} ; \pi \in \mathscr{C}_{1}^{d}, \pi^{\prime} \in \mathscr{U}_{1}^{d}, \sharp \pi^{\prime}=m ;(-1)^{\sharp \pi}\right) \\
= & N\left(s=\pi+m^{2}+\pi^{\prime \prime} ; \pi \in \mathscr{C}_{1}^{d}, \pi^{\prime \prime} \in \mathscr{C}_{1}, l\left(\pi^{\prime \prime}\right) \leqq 2 m ;(-1)^{\sharp \pi}\right) \\
& \text { (here we have merely removed } 1,3,5, \cdots \text { etc. from the } \\
& \text { smallest, next smallest, } \cdots \text { etc. parts of } \pi^{\prime} ; \text { also we use } \\
& \text { the fact that partitions into } \leqq m \text { even parts are equi- } \\
& \text { numerous with partitions into even parts each } \leqq 2 m) \\
= & N\left(s=\pi+\pi^{*}+m^{2}+\pi^{\prime \prime} ; \pi \in \mathscr{G}_{1}^{d}, \pi^{*} \in \mathscr{G}_{2 m+2}^{d}, \pi^{\prime \prime} \in \mathscr{G}_{1},\right. \\
& \left.l\left(\pi^{\prime \prime}\right) \leqq 2 m, l(\pi) \leqq 2 m ;(-1)^{\left.\sharp \pi+\sharp \pi^{*}\right)} \text { (by Lemmas } 1 \text { and } 4\right) \\
= & \left.N\left(s=\pi^{*}+m^{2} ; \pi^{*} \in \mathscr{S}_{2 m+2}^{d} ;(-1)^{\sharp \pi^{*}}\right) \text { (by Lemmas } 1 \text { and } 4\right) .
\end{aligned}
$$

Now the assertion made by the extremes of the above string of equations is valid for $m<0$ also since both sides are identically zero; this is obvious for the left-hand side and follows for the right-hand side by splitting the considered partitions into two equinumerous classes: (1) those in which zero appears and (2) those in which zero does not appear.

Hence if $m$ and $s$ are fixed integers with only $s \geqq 0$ necessarily, then

$$
\begin{aligned}
N(s= & \left.\pi+\pi^{\prime} ; \pi \in \mathscr{S}_{1}^{d}, \pi^{\prime} \in \mathscr{U}_{1}^{d}, \# \pi^{\prime}=m ;(-1)^{\sharp \pi}\right) \\
= & N\left(s=\pi^{*}+m^{2} ; \pi^{*} \in \mathscr{C}_{2 m+2}^{d} ;(-1)^{\sharp \pi^{*}}\right) \\
= & N\left(s=\pi^{\S}+m^{2}+n^{2}+2 m n+n ; \pi^{\S} \in \mathscr{C}_{1}, \sharp \pi^{\S} \leqq n, n \geqq 0 ;\right. \\
& \left.\quad(-1)^{n}\right)
\end{aligned}
$$


(here we have subtracted $2 m+2,2 m+4, \cdots$ etc. from the smallest, next smallest ... etc. parts of $\pi^{*}$ )

$=N\left(s=\pi^{b}+m^{2}+n^{2}+2 m n ; \pi^{b} \in \mathscr{Q}_{1}, \sharp \pi^{b}=n, n \geqq 0 ;(-1)^{\sharp \pi^{b}}\right)$ (here we have added one to each part of $\pi^{\S}$ and included enough ones to get exactly $n$ parts)

$=N\left(s=\pi^{b}+\nu^{2} ; \pi^{b} \in \mathscr{C}_{1}, \nu-\sharp \pi^{b}=m, \nu\right.$ arb. integer; $\left.(-1)^{\sharp \pi^{b}}\right)$.

Each stage of this proof is merely a combinatorial relationship between various types of partitions; however, the actual illustration of each step would be even more cumbersome than with Euler's theorem.

\section{REFERENCES}

1. G.E. Andrews, A simple proof of Jacobi's triple product identity, Proc. Amer. Mat. Soc. 16 (1965), 333-334.

2. — On basic hypergeometric series, mock theta functions, and partitions (I), Quart. J. Math. 17 (1966), 64-80.

3. - On basic hypergeometric series, mock theta functions, and partitions (II), Quart. J. Math. 17 (1966), 132-143.

4. —, q-identities of Auluck, Carlitz, and Rogers, Duke Math. J. 33 (1966), 575582.

5. - Solution to problem 66-9, SIAM Review 9 (1967), 254.

6. - Enumerative proofs of certain q-identities, Glasgow Math. J. 8 (1967), $33-40$.

7. - Note on a partition theorem, Glasgow Math. J. (to appear)

8. — A new property of partitions with applications to the Rogers-Ramanujan identities, J. Comb. Theory, (to appear)

9. G. H. Hardy and E. M. Wright, An Introduction to the theory of numbers, Oxford Unversity Press, Oxford, 4th ed., 1960.

10. L. J. Slater, Generalized hypergeometric functions, Cambridge University Press, Cambridge, 1966.

11. C. Sudler, Two enumerative proofs of an identity of Jacobi, Proc. Edinburgh Math. Soc. (II) 15 (1966), 67-71.

12. J. J. Sylvester, A constructive theory of partitions, Collected papers, IV, Cambridge University Press, Cambridge, 1912.

13. K. T. Vahlen, Beiträge zu einer additiven Zahlentheorie, J. für Math. 112 (1893), $1-36$.

14. E. M. Wright, An enumerative proof of an identity of Jacobi, J. London Math. Soc. 40 (1965), 55-57.

Received May 5, 1969. Partially supported by National Science Foundation Grant GP-8075.

Pennsylvania State University

University Park, Pennsylvania 


\title{
PACIFIC JOURNAL OF MATHEMATICS
}

\author{
EDITORS
}

\author{
H. ROYDEN \\ Stanford University \\ Stanford, California \\ RICHARD PIERCE \\ University of Washington \\ Seattle, Washington 98105
}

\author{
J. DUGUNDJI \\ Department of Mathematics \\ University of Southern California \\ Los Angeles, California 90007 \\ BASIL GORDON \\ University of California \\ Los Angeles, California 90024
}

\section{ASSOCIATE EDITORS}

E. F. BECKENBACH
B. H. NeumanN

F. WOLF
K. YoshidA

\section{SUPPORTING INSTITUTIONS}

\author{
UNIVERSITY OF BRITISH COLUMBIA \\ CALIFORNIA INSTITUTE OF TECHNOLOGY \\ UNIVERSITY OF CALIFORNIA \\ MONTANA STATE UNIVERSITY \\ UNIVERSITY OF NEVADA \\ NEW MEXICO STATE UNIVERSITY \\ OREGON STATE UNIVERSITY \\ UNIVERSITY OF OREGON \\ OSAKA UNIVERSITY \\ UNIVERSITY OF SOUTHERN CALIFORNIA
}

\author{
STANFORD UNIVERSITY \\ UNIVERSITY OF TOKYO \\ UNIVERSITY OF UTAH \\ WASHINGTON STATE UNIVERSITY \\ UNIVERSITY OF WASHINGTON

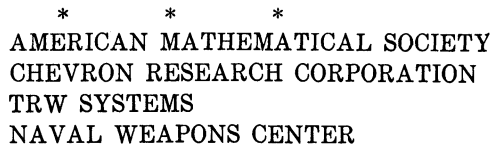

The Supporting Institutions listed above contribute to the cost of publication of this Journal, but they are not owners or publishers and have no responsibility for its content or policies.

Mathematical papers intended for publication in the Pacific Journal of Mathematics should be in typed form or offset-reproduced, double spaced with large margins. Underline Greek letters in red, German in green, and script in blue. The first paragraph or two must be capable of being used separately as a synopsis of the entire paper. It should not contain references to the bibliography. Manuscripts, in duplicate if possible, may be sent to any one of the four editors. Please classify according to the scheme of Math. Rev. 36, 1539-1546. All other communications to the editors should be addressed to the managing editor, Richard Arens, University of California, Los Angeles, California, 90024.

50 reprints are provided free for each article; additional copies may be obtained at cost in multiples of 50 .

The Pacific Journal of Mathematics is published monthly. Effective with Volume 16 the price per volume (3 numbers) is $\$ 8.00$; single issues, $\$ 3.00$. Special price for current issues to individual faculty members of supporting institutions and to individual members of the American Mathematical Society: $\$ 4.00$ per volume; single issues $\$ 1.50$. Back numbers are available.

Subscriptions, orders for back numbers, and changes of address should be sent to Pacific Journal of Mathematics, 103 Highland Boulevard, Berkeley, California, 94708.

PUBLISHED BY PACIFIC JOURNAL OF MATHEMATICS, A NON-PROFIT CORPORATION

Printed at Kokusai Bunken Insatsusha (International Academic Printing Co., Ltd.), 7-17, Fujimi 2-chome, Chiyoda-ku, Tokyo, Japan. 


\section{Pacific Journal of Mathematics}

\section{Vol. 31, No. $3 \quad$ BadMonth, 1969}

George E. Andrews, On a calculus of partition functions .................. 555

Silvio Aurora, A representation theorem for certain connected rings ............ 563

Lawrence Wasson Baggett, A note on groups with finite dual spaces ............. 569

Steven Barry Bank, On majorants for solutions of algebraic differential equations in regions of the complex plane ............................... 573

Klaus R. Bichteler, Locally compact topologies on a group and the corresponding continuous irreducible representations ......................... 583

Mario Borelli, Affine complements of divisors ....................... 595

Carlos Jorge Do Rego Borges, A study of absolute extensor spaces ............. 609

Bruce Langworthy Chalmers, Subspace kernels and minimum problems in Hilbert

spaces with kernel function ...................................... 619

John Dauns, Representation of L-groups and F-rings................. 629

Spencer Ernest Dickson and Kent Ralph Fuller, Algebras for which every

indecomposable right module is invariant in its injective envelope ...........

Robert Fraser and Sam Bernard Nadler, Jr., Sequences of contractive maps and fixed

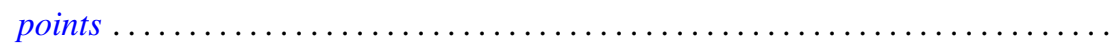

Judith Lee Gersting, A rate of growth criterion for universality of regressive

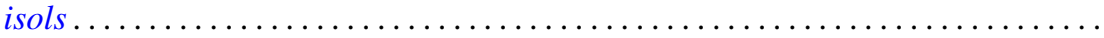

Robert Fred Gordon, Rings in which minimal left ideals are projective ............

Fred Gross, Entire functions of several variables with algebraic derivatives at certain algebraic points

W. Charles (Wilbur) Holland Jr. and Stephen H. McCleary, Wreath products of ordered permutation groups .........................

W. J. Kim, The Schwarzian derivative and multivalence .................. 717

Robert Hamor La Grange, Jr., On $(\mathrm{m}-\mathrm{n})$ products of Boolean algebras ......... 725

Charles D. Masiello, The average of a gauge ........................ 733

Stephen H. McCleary, The closed prime subgroups of certain ordered permutation

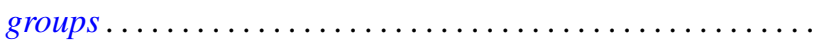

Richard Roy Miller, Gleason parts and Choquet boundary points in convolution measure algebras ...............................

Harold L. Peterson, Jr., On dyadic subspaces ........................ 773

Derek J. S. Robinson, Groups which are minimal with respect to normality being intransitive........................................... 777

Ralph Edwin Showalter, Partial differential equations of Sobolev-Galpern type . . . 787

David Slepian, The content of some extreme simplexes ................... 795

Joseph L. Taylor, Noncommutative convolution measure algebras ............. 809

B. S. Yadav, Contractions of functions and their Fourier series ............... 827

Lindsay Nathan Childs and Frank Rimi DeMeyer, Correction to automorphisms of separable algebras" ....................... 833

Moses Glasner and Richard Emanuel Katz, Correction to: "Function-theoretic degeneracy criteria for Riemannian manifolds".............

Satish Shirali, Correction to: "On the Jordan structure of complex Banach

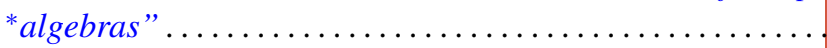

